\title{
ENERgy-EFFicient PATH Configuration METHOD FOR DEF IN WSNS
}

\author{
Jae Kwon Lee, Su Man Nam and Tae Ho Cho \\ School of Information and Communication Engineering Sungkyunkwan University, Su-won, \\ 440-476, Republic of Korea
}

\begin{abstract}
In wireless sensor networks (WSNs), sensor nodes have stringent energy and computation requirements. Since they are left unattended, an adversary can generate false report attacks through compromised nodes. This would result in an increased number of verifications to filter these reports, causing waste of scarce energy resources. In this paper, we propose energy-efficient path configuration method for dynamic enroute filtering $(E D E F)$, which uses fuzzy logic to select the forwarding node(s). In order to cater to compromised nodes and to detect and filter the false reports, we create alternative paths. Our proposed method selects forwarding nodes based on the key content of neighbor nodes, the number of neighbor nodes, and the residual energy. Auth-keys are distributed randomly from the global key pool. Simulation results demonstrate the effectiveness of our proposed method with up to $8.2 \%$ energy efficiency as compared to the dynamic en-route filtering (DEF) scheme.
\end{abstract}

\section{KEYWORDS}

Sensor network, en-route filtering, false report attack, fuzzy logic, detection power, next-hop, energyefficiency

\section{INTRODUCTION}

Wireless sensor networks (WSNs) are being used in numerous applications to carry out tasks without human intervention [1]. However, WSNs suffer energy and computation limitations and are left unattended for longer periods of time [2,3]. Sensor nodes can be compromised and used by an adversary to generate false report attacks. These false report attacks would result in an increased number of verifications to filter these reports, thereby causing a reduced networklifetime [4]. The dynamic en-route filtering (DEF) was proposed by $\mathrm{Yu}$ et al. to defend a false report injection attack [5]. Various schemes as the statistical en-route filtering (SEF), commutative cipher based en-route filtering (CCEF) and probabilistic voting-based filtering (PVFS) as well as the DEF were proposed for the same purpose [6-15]. However, the DEF is capable of performing best in a dynamic network.

The DEF is basically configured as a cluster-based network. The DEF is divided into three major phases: key pre-distribution, key dissemination, and report forwarding. The key pre-distribution phase is executed when the sensor network is first set up. The key dissemination phase is executed whenever the network topology is changed. The report forwarding phase is executed whenever a report is generated by a cluster-head $(\mathrm{CH})$. The DEF verifies the validity of a report using a message authentication code (MAC). To select the next-hop forwarding node, the DEF does not specify the selection criteria which can be changed by applications. In the proposed method, we use the key content of neighbor nodes, the number of neighbor nodes, and the 
residual energy as criteria for the selection of forwarding nodes. The major contributions of our scheme are as follows:

- Early false report detection power

- Considerable energy improvement over the original scheme

- Fuzzy logic for evaluation of the next-hop

In this paper, in order to use next-hop forwarding nodes, we use the above selection criteria with fuzzy logic in our implementation. Whenever the network topology changes, criteria for the selection of forwarding nodes is applied at the key distribution phase. The parameters of the fuzzy input are as follows: the node with multiple keys, the number of neighbor nodes, and the residual energy. We determine the next-hop forwarding node through these input values. Any time the topology of a network is changed, the energy-efficient path configuration method for DEF (EDEF) executes the key dissemination phase, similar to the DEF for a dynamic network. Whenever the key dissemination phase is performed, the next-hop node is selected through fuzzy logic in the EDEF.

The EDEF was organized as a cluster-based sensor network similar to DEF. The cluster-based network can avoid redundant data transmission [16, 17]. A base station (BS) calculates a fuzzy value for each node since the sensor network information is handled by the BS. Each $\mathrm{CH}$ determines the forwarding node through the fuzzy values of the neighbor nodes. Using this information, we demonstrate the EDEF, which results in greater energy efficiency and detection power in comparison with the DEF.

This paper is organized as follows: in a section 2, we describe the original DEF in detail. In section 3, we explain the motivation, overview, and the proposed EDEF in detail. Section 4 discusses assumptions and the performance comparison; and the conclusion is given at the end of the paper.

\section{BACKGROUND}

In this section, we present related background work.

\subsection{Dynamic En-route Filtering Scheme}

The DEF was proposed by Yu et al. to defend the false report injection attack in WSNs. The DEF is composed of a cluster, as in Figure 1, where the nodes v1 v4 represent a sensor node in the cluster, and a black dot (v3) represents a compromised node. Basically, a $\mathrm{CH}$ aggregates the detected reports of all nodes. The $\mathrm{CH}$ then generates a message to forward to the BS. Finally, the $\mathrm{CH}$ sends the message to the BS. Any can be compromised node, including the $\mathrm{CH}$. Additionally, all nodes can be a forwarding node on a path of the BS.

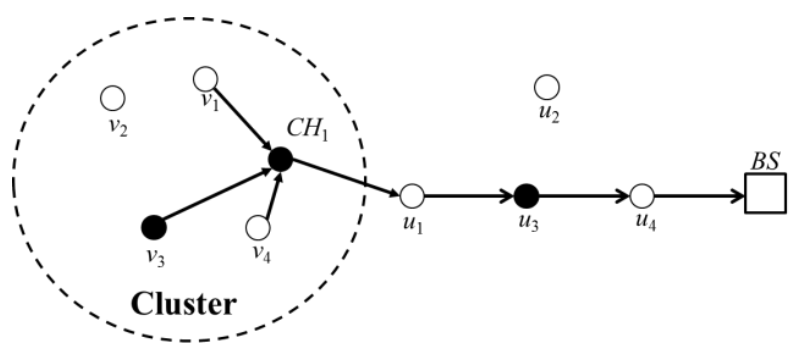

Figure 1. A simple figure of the DEF 
DEF is briefly divided into three phases (key pre-distribution, key dissemination, and report forwarding) as shown in Figure 2. First, the network is configured and then a key is predistributed. The key pre-distribution phase is executed once when the network is first configured. Following this, the key dissemination phase of a key distribution occurs, which executes the report forwarding phase. The key dissemination phase would then be executed again based on the fixed period that is determined at the cluster. The report forwarding phase could be executed at every round at the each forwarding node.

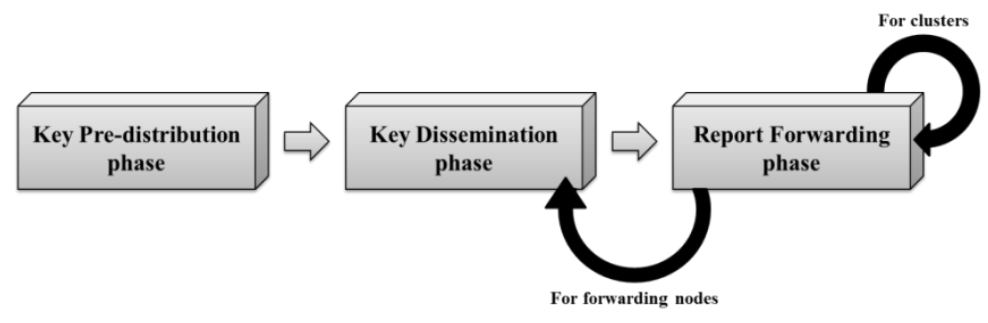

Figure 2. Three phases of the DEF

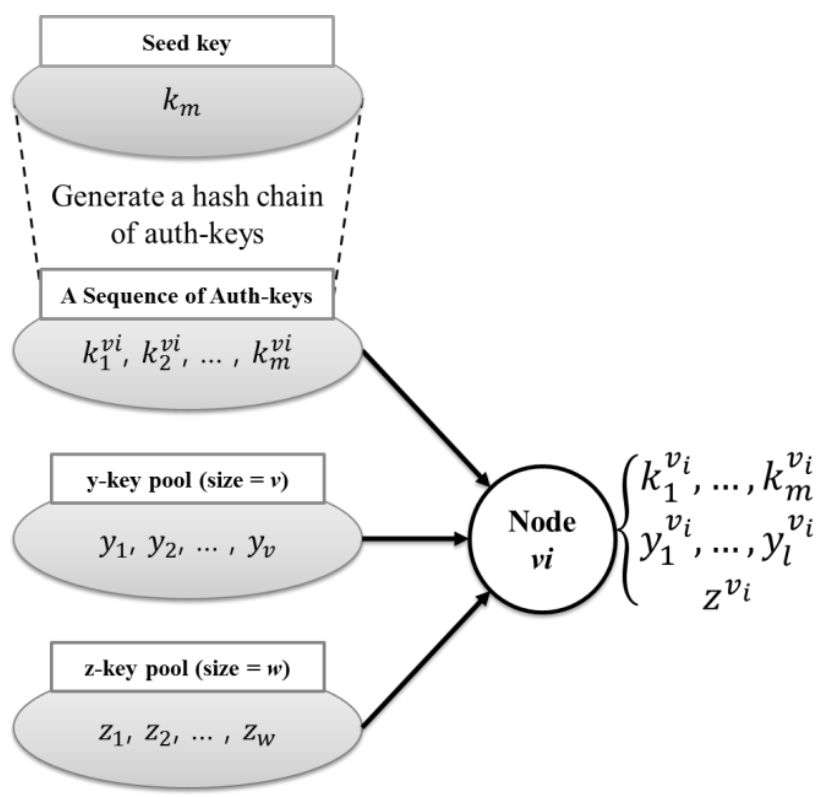

Figure 3. Key deployment of each node

Figure 3 shows that node $v_{-}$i was loaded with the keys in the key pre-distribution phase. Here, the auth-key chain is generated using the seed key and a hash function. This auth-key is then the first of the auth-key chain. The y-key would then be randomly selected as a fixed number in the global key pool. The z-key is then randomly selected as one key of the global key pool; this key is not duplicated with any other keys. The auth-keys of the node in a cluster are aggregated with the $\mathrm{CH}$ to generate the message, $\mathrm{K}(\mathrm{n})$, as in Figure 4. Then the $\mathrm{CH}$ sends the message to q number of forwarding nodes. In the report forwarding phase, a report is aggregated from a node of the cluster as in the key dissemination phase, and then the $\mathrm{CH}$ sends an aggregating message to the BS. In the DEF, the report is verified with the MAC. This MAC is generated using the event and auth-key in each node. The DEF re-executes the key dissemination phase and the report forwarding phase according to a fixed rule. 


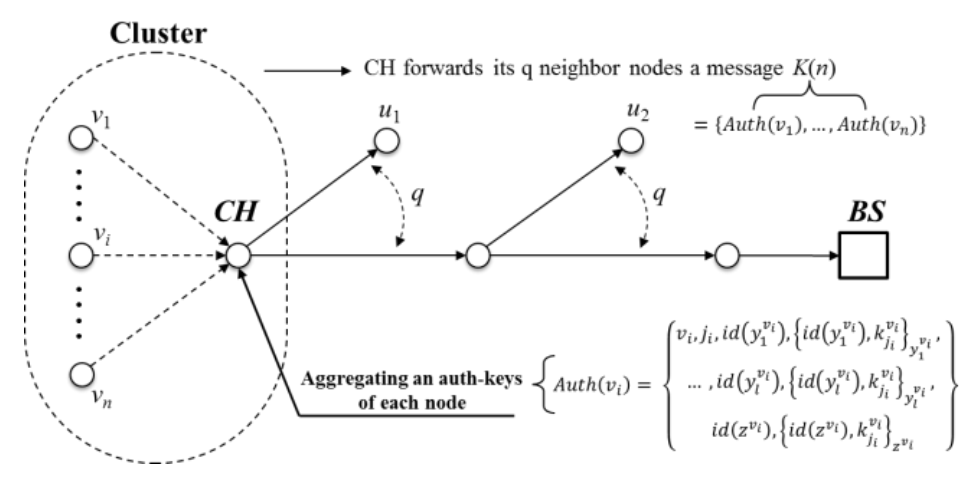

Figure 4. Auth-key is sent to the forwarding node.

The report forwarding phase is newly executed by the forwarding node whenever an event is generated. The report forwarding phase is executed to forward a newly generated event in report format to the BS. The key dissemination phase is re-executed for key re-dissemination in case of network topology changes as such changes can change the nodes of the cluster. It is then necessary to re-disseminate the node information over the changed cluster. Therefore, the key dissemination phase can be re-executed.

\section{Proposed Method}

\subsection{Motivation}

When we select the next forwarding node, the DEF does not specify some factors because, according to various applications, the determining factors may be different. The DEF selects the next node by combining factors such as the available of energy, the link quality, the speed of energy consumption, and so on. The DEF does not consider the energy-efficiency of the forwarding node. The network, therefore, can easily consume the energy of the node. Energy depletion of a node produces a decrease in the network-lifetime. We evaluated the selection of a forwarding node using fuzzy logic; this scheme shows efficiency up to 5\% [18]. However, we decide that the 5\% efficiency is a weak influence. Hence, we changed to new factors and evaluated these factors. According to this, we can have more efficient than before factor through new factors.

\subsection{Overview}

When determining the next forwarding node in the DEF, we select the next node using the fuzzy logic from the proposed EDEF. We used three input factors in fuzzy logic: 1) key content, 2) the number of neighbor nodes, and 3) the residual energy of each candidate node. We can calculate the result using these three input factors. We then determine the next node based on the result. If the report reaches the BS in every round, the BS calculates the result of the nodes in the entire network using fuzzy logic. A CH then determines the next forwarding node on a path to the BS. We can foresee an increase in the efficiency between the EDEF and the DEF through the Travelled hop count of the false reports.

\subsection{Proposed method}

The EDEF uses fuzzy logic in the dissemination phase of the DEF. This is more effective for extending the network-lifetime. The EDEF has three input factors for the fuzzy logic. The three input factors are as follows: 1) the key content $(\mathrm{KN}), 2)$ the number of neighbor node $(\mathrm{NN})$, and 
3) the residual energy (RE) of each contender. When we select the next forwarding node, we use the result from the above three factors. Each input factor is divided into three detailed phases, allowing 27 different possible results $(=3 \times 3 \times 3)$. We can select the optimum forwarding node based on 27 different results. The auth-key is disseminated to multiple paths because of the possibility of 1) data loss, 2) verification, or 3) dynamic topology. The DEF pre-determines the number of forwarding nodes as ' $q$ '. The DEF then selects $q$ as the forwarding node. In case of ' $q$ $=2$,' two forwarding nodes are selected at the BS. When the q possible forwarding nodes are selected, the final forwarding node is chosen using fuzzy logic. Fuzzy rule-based system has been used to approximate reasoning for an imprecision of a data or an uncertainty in reasoning processes [19].

\subsubsection{Further Subsections}

The input factor for fuzzy logic and the resulting value are as follows:

$\mathrm{KN}=\{$ LITTLE, MEDIUM, FULL $\}$

$\mathrm{NN}=\{$ FEW, MIDDLE, CROWDED $\}$

$\mathrm{RE}=\{$ LOW, MEDIUM, ENOUGH $\}$

$\mathrm{R}=\{$ VERY BAD, BAD, NORMAL, GOOD, VERY GOOD $\}$

Each input value is divided into three phases (as shown above), and the resulting value is divided into five parts. Details of the input factors that are used for the fuzzy logic are as follow.

- The key content of node To begin with, the following is the reason for the key content of node. When a key is disseminated in the DEF, a $\mathrm{CH}$ aggregates the keys belonging to each node, and forwards the aggregate to the node on a path in a fixed hop count. We use these keys to verify the forwarded report. At this time, if the node was received in duplicate, the node has multiple keys that are compared with the others. A node with many keys means that it can be used in multiple clusters. According to this, when the DEF sends a report, the node with many keys is more efficient than the others.

- The number of neighbor nodes The number of neighbor nodes is selected as the next input factor so that the message can be delivered to the zones of many candidate nodes. In order to broaden the selection range, the zones of many candidate nodes are selected. If there are few candidate nodes, then the selection pool is reduced. Therefore, we broaden the selection range, in order to obtain a more efficient node.

- The residual energy In the end, the residual energy is the current usable energy of a candidate node and is an input factor. When a node with less energy is compared with a node with a lot of energy, if the node with less energy is selected, the entire network-lifetime is quickly shortened. According to this, if we select the node with a lot of energy, it can sustain the network-lifetime. Selecting the node with a lot of energy can then steadily consume the energy of the whole network. Accordingly, we select it as an input factor for the fuzzy logic.

Figure 5 presents a process in which a $\mathrm{CH}$ selects the $\mathrm{q}$ forwarding node through a fuzzy rulebased system. When the q is determined to be '2,' as in the figure, it receives the respective $\mathrm{KN}$, $\mathrm{NN}$, and RE values of $\mathrm{u}_{-} 1, \mathrm{u}_{-} 2, \llbracket$ and $\mathrm{u} \rrbracket \_3$; it then selects a node based on the two highest ranking results. 


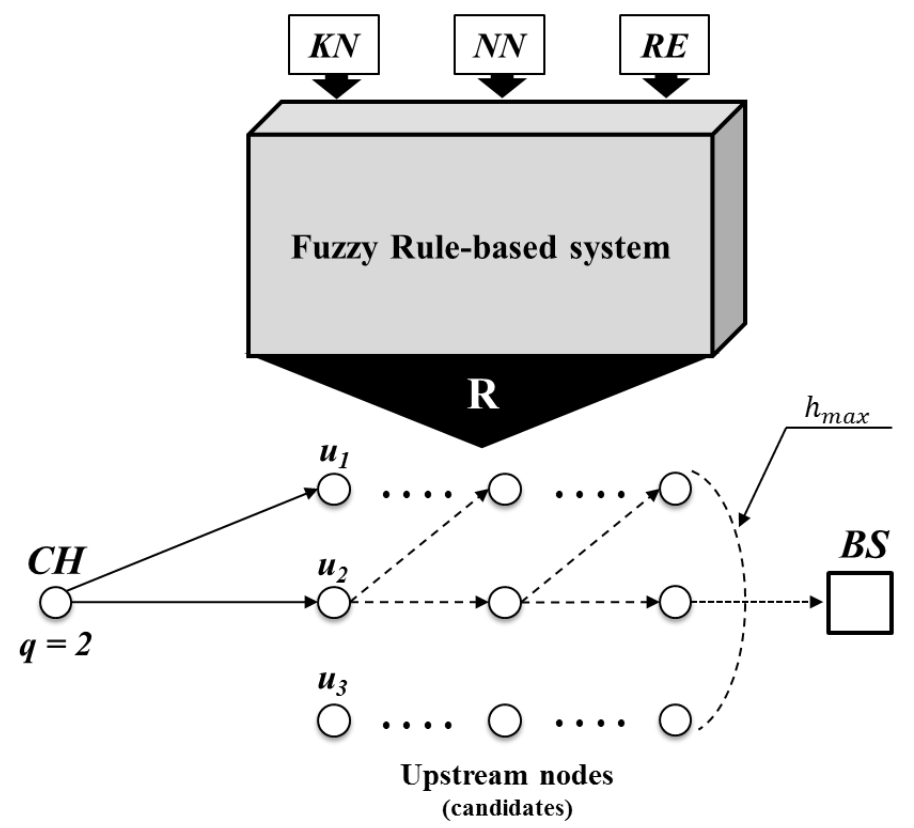

Figure 5. Fuzzy rule-based system for node selection

A fuzzy computation is computed at a BS; the BS has information for all nodes. The BS determines that each $\mathrm{CH}$ selects a node using the fuzzy rule-based system. This is re-computed every cycle in order to forward a message.

Figure 6 presents the membership function of the fuzzy input values and result values.
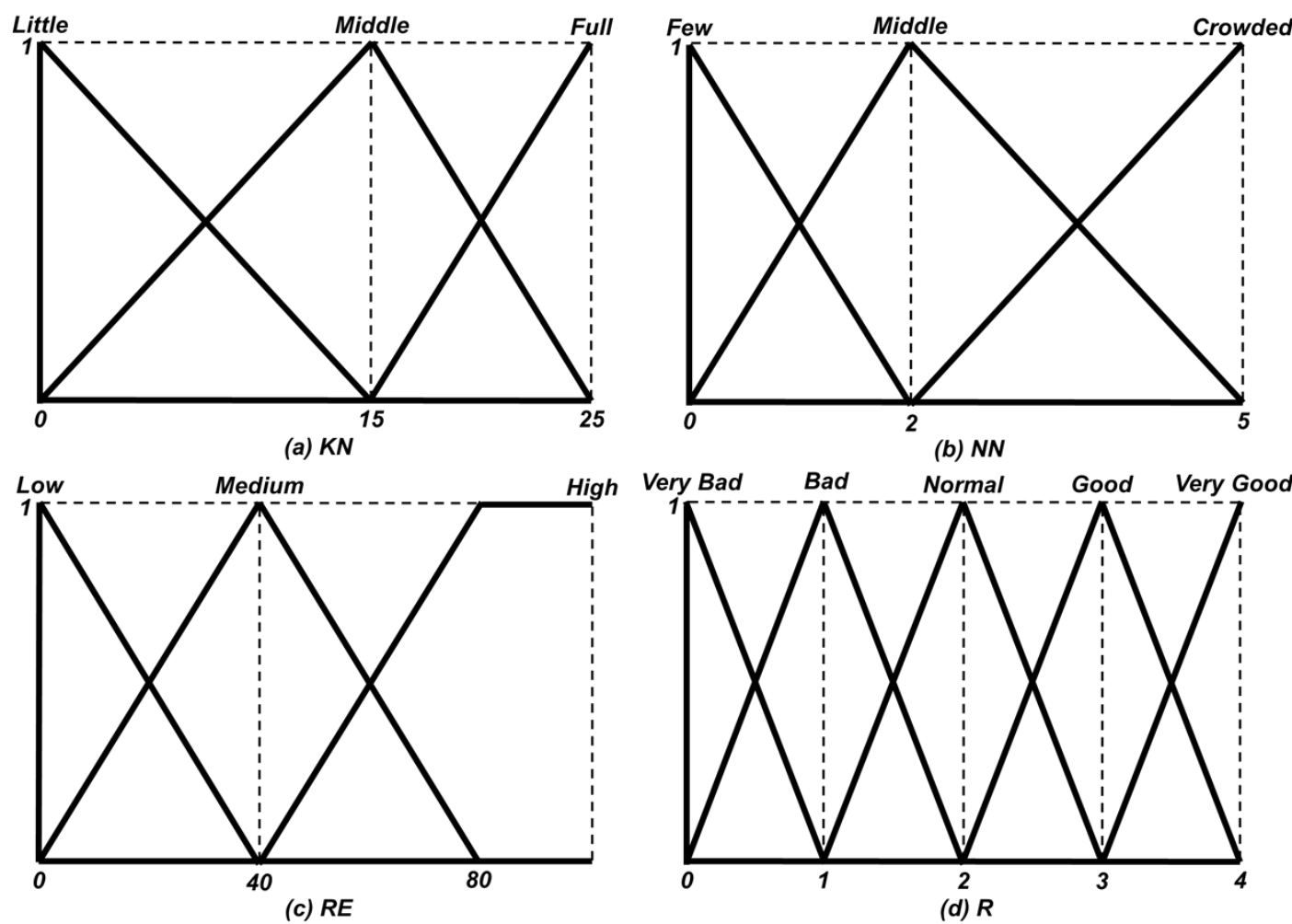

Figure 6. Fuzzy membership function of input values and the result values. 
Some of the rules are shown below.

Table 1. Some of the fuzzy rules.

\begin{tabular}{|c|c|c|c|c|c||c|c|c|c|c|c|}
\hline R1 & $:$ & IF & KN & IS & LITTLE & R5 & $:$ & IF & KN & IS & LITTLE \\
\hline & & AND & NN & IS & FEW & & & AND & NN & IS & FEW \\
\hline & & AND & RE & IS & LOW & & & AND & RE & IS & HIGH \\
\hline & & THEN & R & IS & VERY BAD & & & THEN & R & IS & BAD \\
\hline R2 & $:$ & IF & KN & IS & LITTLE & R6 & $:$ & IF & KN & IS & FULL \\
\hline & & AND & NN & IS & MIDDLE & & & AND & NN & IS & MIDDLE \\
\hline & & AND & RE & IS & LOW & & & AND & RE & IS & MEDIUM \\
\hline & & THEN & R & IS & VERY BAD & & & THEN & R & IS & GOOD \\
\hline R3 & $:$ & IF & KN & IS & LITTLE & R7 & $:$ & IF & KN & IS & FULL \\
\hline & & AND & NN & IS & CROWDED & & & AND & NN & IS & MEDIUM \\
\hline & & AND & RE & IS & LOW & & & AND & RE & IS & LOW \\
\hline & & THEN & R & IS & BAD & & & THEN & R & IS & NORMAL \\
\hline R4 & $:$ & IF & KN & IS & LITTLE & R8 & $:$ & IF & KN & IS & FULL \\
\hline & & AND & NN & IS & FEW & & & AND & NN & IS & MIDDLE \\
\hline & & AND & RE & IS & MEDIUM & & & AND & RE & IS & ENOUGH \\
\hline & & THEN & R & IS & VERY BAD & & & THEN & R & IS & VERY \\
& & & & & & & & & & & GOOD \\
\hline
\end{tabular}

\subsubsection{The base data for the EDEF}

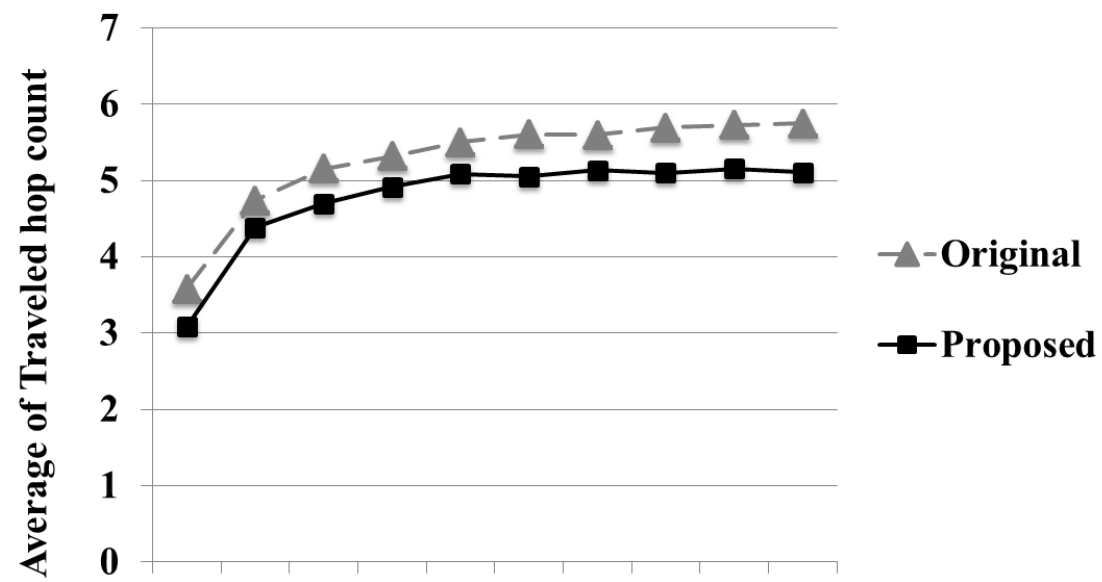

$\begin{array}{llllllllll}10 & 20 & 30 & 40 & 50 & 60 & 70 & 80 & 90 & 100\end{array}$

Attack ratio(\%)

Figure 7. Average of the Travelled hop count for ' $q=3$ '

Figure 7 shows an average of the Travelled hop count for ' $q=3$ ' according to the attack ratio. It is expected that the EDEF can detect a false report earlier than the original DEF. Therefore, we experimented further and obtained the following results. 
International Journal on Information Theory (IJIT),Vol.4, No.2, April 2015

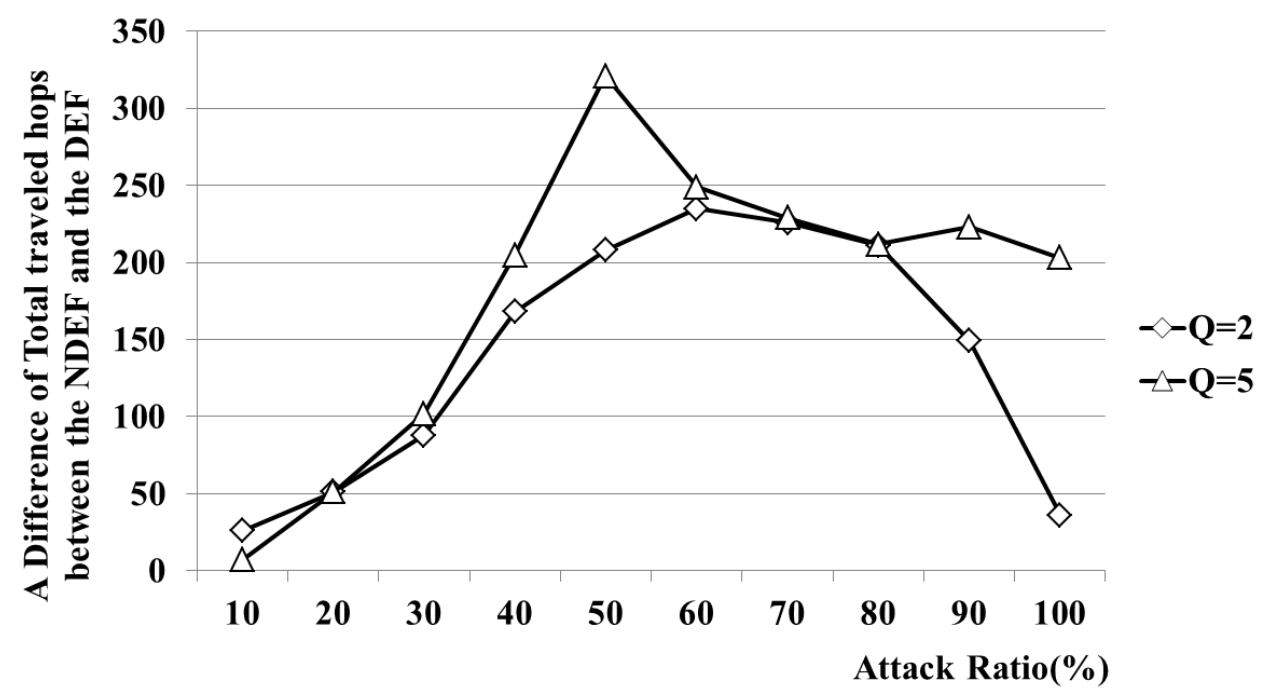

Figure 8. Difference in the total Travelled hops between the EDEF and the DEF.

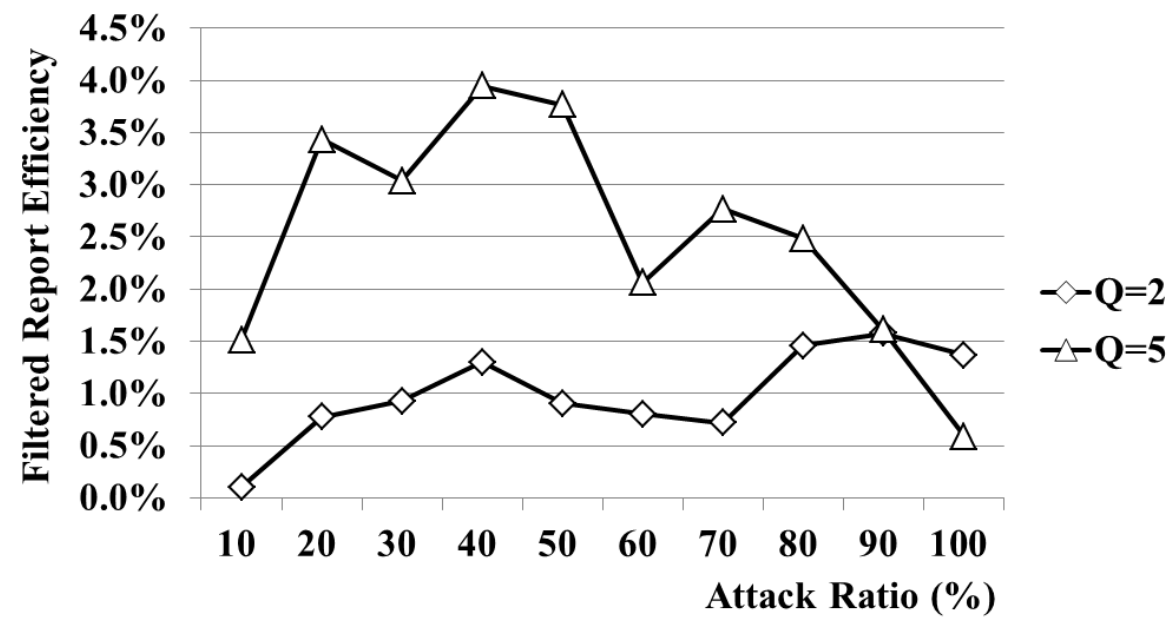

Figure 9. A filtered report ratio

Figure 8 shows the Travelled hop count without filtering a false report. The following is the Travelled hop count equation. The hop count is the difference between the value of the Travelled hop count without filtering using a DEF and the value of the Travelled hop count without filtering using the proposed method.

Total Travelled hops $=$ Travelled hops in the DEF - Travelled hops in the proposed method Additionally, Figure 9 presents a graph of the EDEF efficiency against the DEF. As shown in the figure, greater efficiency is seen with increases in 'q'. According to Figure 8 and 9, the proposed method is faster than the existing method. Accordingly, we progressed to more detailed experiments; in the following section, we verify the energy consumption efficiency compared with the DEF.

\section{Performance Evaluation}

In this section, we will present the energy-efficiency of the EDEF in comparison with the original DEF under same environmental setup. 


\subsection{Experiment setup}

We assume that the BS has information of all nodes in the sensor network. Every $\mathrm{CH}$ has the information about the key content, the number of neighbor nodes, and the residual energy of all nodes in its cluster.

- The sensor field size is $200 \times 200(\mathrm{~m} 2)$, and the total number of nodes are 1000 , consisting of 100 clusters which are randomly distributed where each cluster consists of 10 nodes.

- Each node picks $1=2$ y-keys and one z-key, where the size of the y-key and z-key pool consists of 1000 keys.

- Each node forwards the message $\mathrm{K}(\mathrm{n})$ to q selected downstream neighbor nodes, until $\mathrm{K}(\mathrm{n})$ reaches to the $\mathrm{BS}$ or has been forwarded by hmax hop. Here, hmax $=10$. Hence, we adopted Hill Climbing method [20] to forwards the message K(n).

- Each aggregated report contains $10 \mathrm{MACs}$, and there are at most 10 compromised nodes in each cluster.

- The size of a message is 36 bytes, and $16.25 / 12.5 \mu \mathrm{J}$ is consumed for transmitting/receiving a byte [8].

- Each node has fuzzy result values which are calculated by the BS. The BS then broadcasts the q and fuzzy result of each node to entire network through authenticated broadcast (e.g., using $\mu$ TESLA [21])

- To simulate a dynamic network, we apply a simple ON/OFF operation node, where each node is typically switched to ON or OFF mode.

- The DEF and the EDEF adopted a GPSR routing protocol.

\subsection{Experiment results}

In the above assumption, the announced sensor field size and total number of nodes are the same, and a $\mathrm{CH}$ generates 100 reports during each cycle. Each key pool has 1000 keys. The experiment was performed with an increasing ratio of false reports and a changing q value. The figure below shows the efficiency of the proposed method compared with DEF according to the attack ratio.

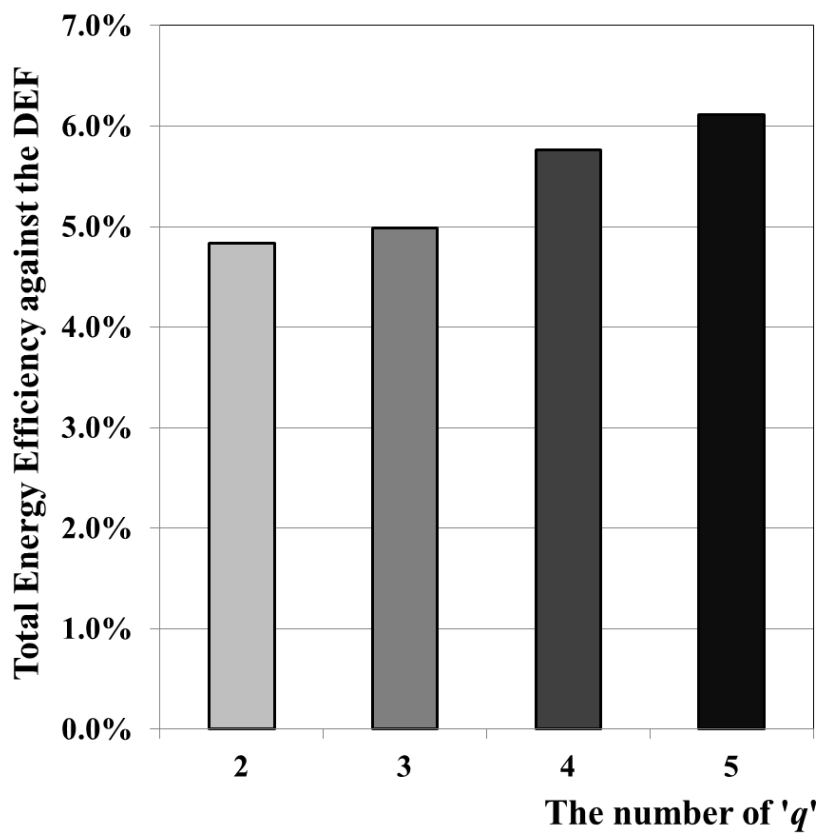

Figure 10. Total energy efficiency of each ' $q$.' 
The figure above shows the energy-efficiency for each ' $q$ ' against the DEF. Each q shown has efficiency between $4.84 \%$ and $6.12 \%$. The greater the number of $\mathrm{q}$ or the more complex the sensor network, the greater is the efficiency. The reason why the efficiency increases as $q$ increases is because a large, $\mathrm{q}$ has a larger number of cases. Therefore, this demonstrates that selection using the fuzzy system is more efficient than the DEF.

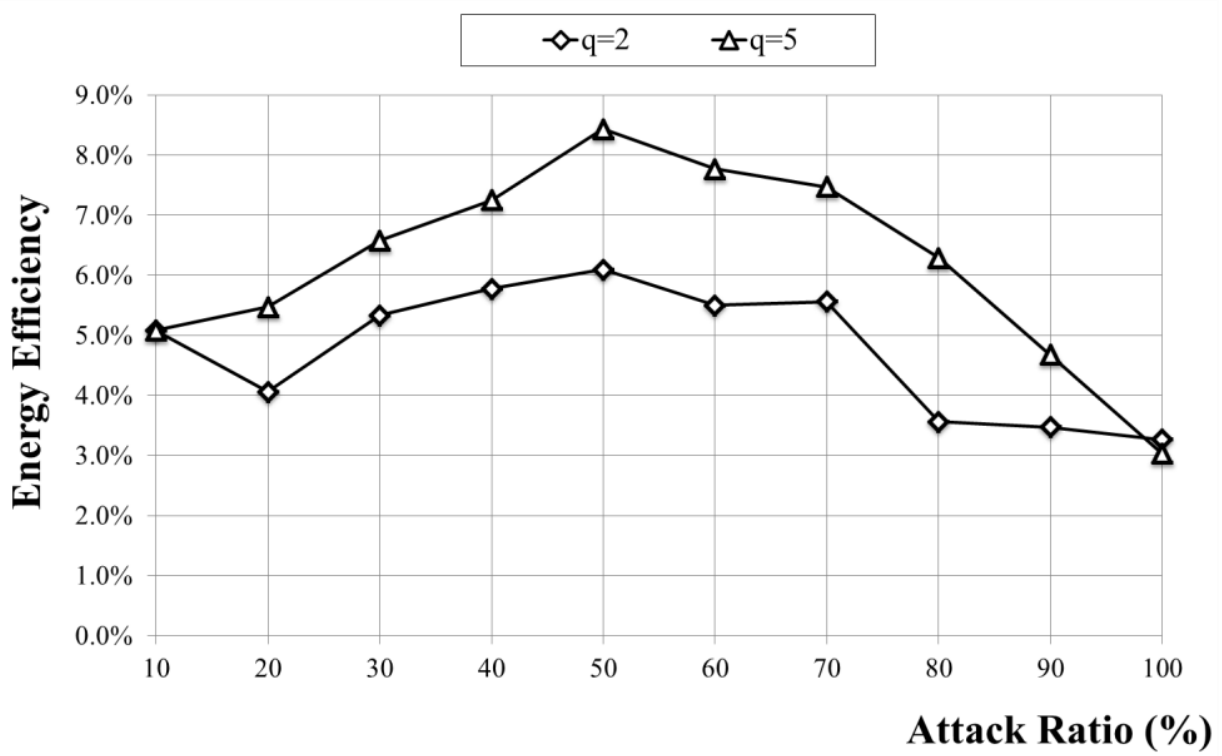

Figure 11. Energy efficiency versus the attack ratio.

By measuring the value for each $\mathrm{q}$, the results show that greater the value of $\mathrm{q}$, the greater the efficiency as compared to the DEF. An increasing value of q means that more messages are created to send to the next forwarding nodes. As can be seen in the above figure, when the value of $\mathrm{q}$ is 5 , the efficiency is greater than $8 \%$. The attack rate of the 100 generated reports is the ratio of false reports. The efficiency ratio of false reports of enemy all the time and when there are many showed similar performance. However, they showed a difference in the $30 \%$ to $70 \%$ section. In fact, a false report injection attack cannot occupy the whole message. The results are confirmed because they were generated for the above experiment. However, the experiment does not include the number of cases in a real sensor network. However, if the number of $q$ is changed according to the environment of the DEF, the proposed method showed greater efficiency through the above experiment.

The figure above compares the efficiency of the proposed method with the DEF with regard to the total number of filtered false reports in one hop. This graph compares the number of filtered reports within one hop in the proposed method with that in the DEF. The figure above shows a slightly more efficient detection for the proposed method compared with the DEF.

\section{Conclusions}

In this study, the aim was to increase energy-efficiency with increased detection power by considering alternative paths. We have presented EDEF, a fuzzy-based next-hop selection scheme. In order to select the forwarding nodes in next-hop and to distribute keys, we use fuzzy logic. Our method can detect more false reports as compared to the DEF scheme. By detecting the false reports early, our method demonstrated considerable energy improvement without compromising the security of the original scheme. 
In conclusion, the EDEF has excellent detection power compared with the DEF and improves the energy efficiency in order to sustain the network-lifetime for a long time. In the future, we can further enhance the performance through a simulation with suitable fuzzy function values.

\section{ACKNOWLEDGEMENTS}

This research was supported by Basic Science Research Program through the National Research Foundation of Korea (NRF) funded by the Ministry of Education, Science and Technology (No. 2013R1A2A2A01013971)

\section{REFERENCES}

[1] I. F. Akyildiz, Weilian Su, Y. Sankarasubramaniam, \& E. Cayirci, (2002) "A survey on sensor networks," Communications Magazine, IEEE, Vol.40, No.8, pp.102-114.

[2] Jing Deng, R. Han, and S. Mishra, (2005) "Countermeasures against traffic analysis attacks in wireless sensor networks," Security and Privacy for Emerging Areas in Communications Networks, pp.113-126.

[3] Moog Inc., "MICAz data sheet," Moog Crossbow, http://www.xbow.com.

[4] C. Karlof and D. Wagner, (2003)"Secure routing in wireless sensor networks: Attacks and countermeasures," Sensor Network Protocols and Applications, 2003. Proceedings of the First IEEE. 2003 IEEE International Workshop on, pp.113-127.

[5] Zhen Yu and Y. Guan, (2010) "A dynamic en-route filtering scheme for data reporting in wireless sensor networks," Networking, IEEE/ACM Transactions on, Vol.18, No.1, pp.150-163.

[6] Hao Yang and Songwu Lu, "Commutative cipher based en-route filtering in wireless sensor networks," Vehicular Technology Conference, 2004. VTC2004-Fall. 2004 IEEE 60th, pp.1223-1227.

[7] Feng Li, Avinash Srinivasan, and Jie Wu, (2008) "PVFS: A probabilistic voting-based filtering scheme in wireless sensor networks," International Journal of Security and Networks, Vol.3, No.3, pp.173-182.

[8] Fan Ye, Haiyun Luo, Songwu Lu, and Lixia Zhang, "Statistical en-route filtering of injected false data in sensor networks," Selected Areas in Communications, IEEE Journal on, Vol.23, No.4, pp.839-850.

[9] Jianzhong Li, Lei Yu, Hong Gao, and Shuguang Xiong, "Grouping-enhanced resilient probabilistic en-route filtering of injected false data in WSNs," Parallel and Distributed Systems, IEEE Transactions on, Vol.23, No.5, pp.881-889.

[10] T. P. Nghiem, Sang Jin Lee, and Tae Ho Cho, (2009) "An energy-aware fuzzy-based en-route filtering scheme in wireless sensor networks," Future Networks, 2009 International Conference on, pp.28-32.

[11] A. S. Uluagac, R. A. Beyah, and J. A. Copeland, (2010) "Time-based DynamiC keying and en-route filtering (TICK) for wireless sensor networks," Global Telecommunications Conference (GLOBECOM 2010), 2010 IEEE, pp.1-6.

[12] Wensheng Zhang and Guohong Cao, (2005) "Group rekeying for filtering false data in sensor networks: A predistribution and local collaboration-based approach," INFOCOM 2005. 24th Annual Joint Conference of the IEEE Computer and Communications Societies. Proceedings IEEE, pp.503514 vol. 1.

[13] Hae Young Lee and Tae Ho Cho, (2006) "Key inheritance-based false data filtering scheme in wireless sensor networks," Distributed Computing and Internet Technology, Lecture Notes in Computer Science, vol.4317, pp.116-127.

[14] Hae Young Lee and Tae Ho Cho, (2008) "Fuzzy logic based key disseminating in ubiquitous sensor networks," Advanced Communication Technology, 2008. ICACT 2008. 10th International Conference on, pp.958-962.

[15] Ting Yuan, Shiyong Zhang, Yi-ping Zhong, and Jianqing Ma, (2008) "KAEF: An en-route scheme of filtering false data in wireless sensor networks," Performance, Computing and Communications Conference, 2008. IPCCC 2008. IEEE International, pp.193-200.

[16] J. N. Al-Karaki and A. E. Kamal, (2004) "Routing techniques in wireless sensor networks: A survey," Wireless Communications, IEEE, vol.11, no.6, pp.6-28.

[17] K. Akkaya and M. Younis, (2005) "A survey on routing protocols for wireless sensor networks," $\mathrm{Ad}$ Hoc Networks, vol.3, no.3, pp.325-349, 5. 
[18] Jae Kwon Lee and Tae Ho Cho, (2012) "Selecting next hop nodes for energy efficiency in dynamic filtering based WSNs using fuzzy system," Information Science and Digital Content Technology (ICIDT), 2012 8th International Conference on, pp.674-677.

[19] H. S. N. Serrano, (2007) "Landing site selection using fuzzy rule-based reasoning," Proc. ICRA, pp.4899-4904.

[20] D.B. Skalak, (1994) "Prototype and feature selection by sampling and random mutation hill climbing algorithms," In Proc. of Eleventh International Machine Learning, pp.293-301, 1994.

[21] Adrian Perrig, Robert Szewczyk, J. D. Tygar, Victor Wen, and David E. Culler, (2002) "SPINS: Security protocols for sensor networks," Wireless Networks, Vol.8, No.5, pp.521-534.

[22] Lee, S.hyun. \& Kim Mi Na, (2008) "This is my paper”, ABC Transactions on ECE, Vol. 10, No. 5, pp120-122.

[23] Gizem, Aksahya \& Ayese, Ozcan (2009) Coomunications \& Networks, Network Books, ABC Publishers.

\section{Authors}

Jae Kwon Lee received his B.S. degree in Computer Software Engineering from the Kumoh National Institute of Technology, Republic of Korea, in 2012, M.S degrees in Electrical and Computer Engineering from Sungkyunkwan University in 2014, respectively. His research interests are in the areas of wireless sensor network, intelligent systems, modelling \& simulation, and enterprise resource planning.

Su Man Nam received his B.S. degrees in computer information from Hanseo University, Korea, in February 2009 and M.S degrees in Electrical and Computer Engineering from Sungkyunkwan University in 2013, respectively. He is currently a doctoral student in the College of Information and Communication Engineering at Sungkyunkwan University, Korea. His research interests include wireless sensor network, security in wireless sensor networks, and modelling \& simulation.
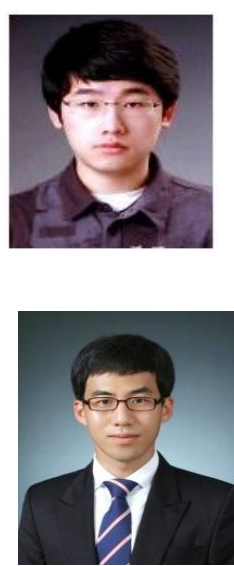

Tae Ho Cho received the Ph.D. degree in Electrical and Computer Engineering from the University of Arizona, USA, in 1993, and the B.S. and M.S. degrees in Electrical Engineering from Sungkyunkwan University, Republic of Korea, and the University of Alabama, USA, respectively. He is currently a Professor in the College of Information and Communication Engineering, Sungkyunkwan University, Korea. His research interests are in the areas of wireless sensor network, intelligent systems, modeling \& simulation, and enterprise resource planning.

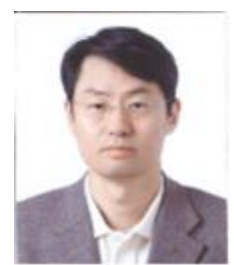

